\title{
Pertinent choice of parameters for discrete Kautz approximation
}

\author{
N. Tanguy, R. Morvan, P. Vilbé, and L.C. Calvez ${ }^{1}$
}

\begin{abstract}
Kautz functions have received much attention in the recent mathematical modeling and identification literature. These functions which involve free parameters can approximate efficiently signals with strong oscillatory behavior. We consider here the choice of the free parameters in discrete (two-parameter) Kautz approximation. Using a key relationship between Kautz and Laguerre expansions we derive an upper bound for the quadratic truncation error. Minimization of this upper bound yields pertinent parameters, whose computation then requires reduced knowledge of the function to be modeled.
\end{abstract}

Index terms: Orthonormal approximation, Kautz functions, Modeling.

\section{INTRODUCTION}

In recent years there has been large interest in the use of discrete Laguerre functions in approximation, modeling, filtering, identification and control. Nevertheless, poorly damped systems are difficult to approximate with a reasonable number of Laguerre functions, so the so-called two-parameter discrete Kautz functions, which can approximate more efficiently signals with strong oscillatory behavior, have received much attention in the recent mathematical modeling and identification literature (see, e.g., [1], [2], [3]). These functions, which involve free parameters, form complete orthonormal sets in $\ell^{2}[0, \infty)$. In practice, truncated series will be used. For a large number of expansion terms the choice of the free parameters is not crucial but for a limited number of expansion terms the choice of the parameters is then of importance to provide a good approximation to a given function. This question has already been answered for discrete- and continuous-time Laguerre-functions. Scientists have investigated two different approaches. In the first one, the error energy is minimized; in the particular case of Laguerre functions, for example, Masnadi-Shirazi and Ahmed [4], [5], [6] have developed an analytical approach that requires finding roots of possibly high-order polynomials. Reports have dealt with similar results obtained in an optimal pole position of Laguerre filters with arbitrary inputs [7] and [8]. Later, the optimality conditions for truncated Kautz series and networks involving two parameters have been presented [9], [10]. Such an approach generally entails an excessive computational cost. The alternative approach consists of minimizing an upper bound for the error energy. The case of continuous-time Laguerre functions has been previously studied in [11] and [12]. More recently, a suboptimal procedure to determine the poles of discrete-time Laguerre functions was presented [13], [14]. This last technique has the great merit that the derivation of a solution requires knowledge of only few numerical characteristics of the signal under consideration. It has been generalized to a class of orthogonal functions, which satisfy a suitable differential or difference equation [15], [16], [17]. Unfortunately, the required differential or difference equation relative to continuous- and discrete-time Kautz functions haven't been established and the technique can't be used. In [18] we have studied the choice of the parameters in continuous-time Kautz approximations. We used a particular transformation relating Kautz functions to Laguerre functions that allows us to deduce a suboptimal but easy-to-use procedure to choose the free parameters. In this correspondence we deal with the discrete-time case, and propose a useful technique to compute the required numerical characteristics of the signal under consideration. The correspondence is organized as follows. We remind of the discrete-time Kautz functions and present the key relationship between Kautz expansion coefficients and Laguerre series in Section II. In section III a procedure to choose the free parameters in discrete Kautz approximation is deduced. A useful technique to compute the required moments of the function to be modeled is given in Section IV. In Section V we present an illustrative example before concluding in Section VI.

\section{KAUTZ FUNCTIONS AND KEY RELATIONSHIP}

The discrete Kautz functions $\psi_{l}(k, b, c)$ can be defined by their z-transforms

$$
\begin{aligned}
\Psi_{2 n}(z) & =\frac{z \sqrt{\left(1-c^{2}\right)\left(1-b^{2}\right)}}{z^{2}+b(c-1) z-c}\left(\frac{-c z^{2}+b(c-1) z+1}{z^{2}+b(c-1) z-c}\right)^{n} \\
\Psi_{2 n+1}(z) & =\frac{z(z-b) \sqrt{1-c^{2}}}{z^{2}+b(c-1) z-c}\left(\frac{-c z^{2}+b(c-1) z+1}{z^{2}+b(c-1) z-c}\right)^{n} \\
-1 & <b<1,-1<c<1, n=0,1,2, \ldots
\end{aligned}
$$

${ }^{1}$ Laboratoire d'Electronique et des Systèmes de Télécommunications (LEST), FRE CNRS n²269, Université de Bretagne Occidendale (UBO), 29285 BREST cedex, FRANCE. \{Noel.Tanguy, Riwal.Morvan, Pierre.Vilbe, Leon-Claude.Calvez\}@univ-brest.fr 
with $l=2 n$ or $l=2 n+1$ and where the numbering of the functions as defined in [2] has been slightly modified for suitability. The parametrization of $\psi_{l}(k, b, c)$ are particular cases of the so-called normalized ladder filters [19] with $b$ and $c$ denoting the k-parameters.

The discrete-time functions are written as $\psi_{l}(k)$ or as $\psi_{l}(k, b, c)$ whenever it is desirable to exhibit the parameters. The orthonormal set $\left\{\psi_{l}\right\}$ is complete in $\ell^{2}[0, \infty)$, thus any finite energy real causal signal $f(k)$ can be approximated within any prescribed accuracy by truncating its infinite expansion $f(k)=\sum_{l=0}^{\infty} a_{l} \psi_{l}(k)$ where $a_{l}=\left\langle f, \psi_{l}\right\rangle$ is the $l+1$ th Fourier coefficient. The $(L=2 N)$-term truncated expansion yields the best approximation to $f(k)$ of the form $\widetilde{f}(k)=\sum_{l=0}^{L-1} a_{l} \psi_{l}(k)$ in the sense of minimizing the quadratic (truncation) error

$$
I(f) \hat{=} \sum_{k=0}^{\infty}[f(k)-\tilde{f}(k)]^{2}=\|f\|^{2}-\sum_{l=0}^{L-1} a_{l}^{2}=\sum_{l=L}^{\infty} a_{l}^{2} .
$$

Usually, since the $a_{l}$ depend on $b$ and $c, I(f)$ can be reduced further by a proper choice of these parameters. Nice optimality conditions for Kautz approximation, generalizing that of the Laguerre case, have been derived in [10]. However, these conditions of great theoretical interest can result in complicated computations in practical cases. For Laguerre functions [13] and other classical functions [15], [16] an alternative easy-to-use and efficient approach, based on minimization of an upper bound of the error energy, has been proposed. In the following we derive a somewhat similar procedure for the specific set of non-classical two-parameter discrete Kautz functions.

Recently [2], it has been shown that the coefficients $a_{l}$ can be found from power series calculations related to Laguerre functions defined by

$$
\Phi_{n}(z, c)=\sqrt{1-c^{2}} \frac{z}{z-c}\left(\frac{1-c z}{z-c}\right)^{n}
$$

where $\Phi_{n}(z, c)$ are the z-transforms of discrete-time Laguerre functions $\phi_{n}(k, c)$ which, like the Kautz functions, form a complete orthonormal set in $\ell^{2}[0, \infty)$.

Now, let us define

$$
P(z)=\frac{z(z-b)}{1-b z}, \quad \text { and } \quad Q(z)=-\frac{z-b}{1-b z} .
$$

Note that $P(Q(z))=P(z)$ and that $P(1 / z)=1 / P(z)$. We also have

$$
\Psi_{2 n+1}(z)=\Phi_{n}(P(z), c)
$$

and from the definition of the Kautz functions (1)

$$
\Psi_{2 n}(z)=\frac{\sqrt{1-b^{2}}}{z-b} \Psi_{2 n+1}(z) .
$$

Let

$$
G_{e}(z) \widehat{=} \sum_{n=0}^{\infty} a_{2 n} \Phi_{n}(z, c)
$$

and

$$
G_{o}(z) \widehat{=} \sum_{n=0}^{\infty} a_{2 n+1} \Phi_{n}(z, c) .
$$

With these definitions and the properties of $P(z)$ and $Q(z)$, we have

$$
F_{e}(z) \widehat{=} G_{e}(P(z))=\sum_{n=0}^{\infty} a_{2 n} \Psi_{2 n+1}(z)=F_{e}(Q(z))
$$

and

$$
F_{o}(z) \widehat{=} G_{o}(P(z))=\sum_{n=0}^{\infty} a_{2 n+1} \Psi_{2 n+1}(z)=F_{o}(Q(z))
$$

Denoting by $F(z)$ the z-transform of $f(k)$,

$$
F(z)=\sum_{n=0}^{\infty}\left[a_{2 n} \Psi_{2 n}(z)+a_{2 n+1} \Psi_{2 n+1}(z)\right]
$$


where $F(z)$ is assumed to be analytic outside the unit circle in the $z$-plane, it follows that

$$
F(z)=F_{o}(z)+\frac{\sqrt{1-b^{2}}}{z-b} F_{e}(z)
$$

and

$$
F(Q(z))=F_{o}(z)-\frac{1-b z}{z \sqrt{1-b^{2}}} F_{e}(z)
$$

from which one can deduce

$$
\begin{aligned}
G_{e}\left(\frac{z(z-b)}{1-b z}\right) & =F_{e}(z) \\
& =\frac{\sqrt{1-b^{2}} z(z-b)}{-b z^{2}+2 z-b}\left[F(z)-F\left(-\frac{z-b}{1-b z}\right)\right]
\end{aligned}
$$

and

$$
G_{o}\left(\frac{z(z-b)}{1-b z}\right)=F_{o}(z)=\frac{1}{-b z^{2}+2 z-b}\left[(z-b)(1-b z) F(z)+z\left(1-b^{2}\right) F\left(-\frac{z-b}{1-b z}\right)\right]
$$

Setting $\frac{z(z-b)}{1-b z}=u$ yields $z=\xi_{ \pm}(u) \hat{=}\left[b(1-u) \pm \sqrt{b^{2}(1-u)^{2}+4 u}\right] / 2$, thus substituting $\xi_{ \pm}(z)$ for $z$ in $(6)$ and $(7)$ will give $G_{e}(z)$ and $G_{o}(z)$. Notice that the choice of the sign is not a problem since the plus sign and the minus sign yield the same result for $G_{e}(z)$ and $G_{o}(z)$. Thus using (4) and (5) the coefficients $a_{2 n}$ and $a_{2 n+1}$ associated with the expansion of $f(k)$ with respect to the orthonormal set $\left\{\psi_{l}\right\}$ are readily obtained from $G_{e}(z)$ and $G_{o}(z)$.

\section{PROPOSED PROCEDURE FOR PERTINENT PARAMETERS}

From [15] it is known that, provided $N \geq\left[\left(M_{1}(x)+M_{0}(x)\right) c^{2}-2 c M_{2}(x)+M_{1}(x)\right] /\left[\left(1-c^{2}\right) M_{0}(x)\right]$, the squared error $Q(x)=\sum_{n=N}^{\infty} a_{n}^{2}$ of a truncated Laguerre series expansion $\widetilde{x}(k)=\sum_{n=0}^{N-1} a_{n} \phi_{n}(k, c)$ of a discrete-time signal $x(k)$ is bounded by

$$
Q(x) \leq B(c ; x)=\frac{\left(M_{1}(x)+M_{0}(x)\right) c^{2}-2 c M_{2}(x)+M_{1}(x)}{N\left(1-c^{2}\right)}
$$

where

$$
\begin{aligned}
& M_{0}(x)=\sum_{k=0}^{\infty} x^{2}(k)=\|x\|^{2}, \\
& M_{1}(x)=\sum_{k=0}^{\infty} k x^{2}(k)
\end{aligned}
$$

and

$$
M_{2}(x)=\sum_{k=0}^{\infty} k x(k) x(k-1)
$$

Denoting by $g_{e}(k, b)$ and $g_{o}(k, b)$ the inverse z-transforms of $G_{e}(z)$ and $G_{o}(z)$, and applying this result to the approximation of $g_{e}(k, b)$ and $g_{o}(k, b)$ (see $(4)$ and $\left.(5)\right)$ it follows that

$$
Q\left(g_{i}\right) \leq B\left(c ; g_{i}\right)=\frac{\left(M_{1}\left(g_{i}\right)+M_{0}\left(g_{i}\right)\right) c^{2}-2 c M_{2}\left(g_{i}\right)+M_{1}\left(g_{i}\right)}{N\left(1-c^{2}\right)}
$$

where $i=e$ or $o$ and provided that $N \geq\left[\left(M_{1}\left(g_{i}\right)+M_{0}\left(g_{i}\right)\right) c^{2}-2 c M_{2}\left(g_{i}\right)+M_{1}\left(g_{i}\right)\right] /\left[\left(1-c^{2}\right) M_{0}\left(g_{i}\right)\right]$, a condition which is assumed to hold in the following ( $N$ is sufficiently large). The quadratic error of the Kautz approximation of $f(k)$ is related to the quadratic error of the Laguerre approximation of $g_{o}(k)$ and $g_{e}(k)$ by $I(f)=Q\left(g_{o}\right)+Q\left(g_{e}\right)^{2}$. Dividing $I(f)$ throughout by $\|f\|^{2}=M_{0}\left(g_{e}\right)+M_{0}\left(g_{o}\right)$, the normalized quadratic error associated with a $(L=2 N)$-term Kautz approximation of $f(k)$ is then bounded by

$$
q \leq B=\frac{\left(m_{1}+1\right) c^{2}-2 m_{2} c+m_{1}}{N\left(1-c^{2}\right)} .
$$

\footnotetext{
${ }^{2}$ This idea can be extended to the determination on an upper bound for the summation of the squared error of the simultaneous approxi-
} mation of several $f(k)$ functions. 
where $q \widehat{=} I(f) /\|f\|^{2}, m_{j}(b) \widehat{=}\left[M_{j}\left(g_{e}\right)+M_{j}\left(g_{o}\right)\right] /\|f\|^{2}, j=1,2$. This bound attains its minimum when

$$
\left\{\begin{array}{lll}
c=c_{0}=\rho-\sqrt{\rho^{2}-1} & \text { if } & \rho>1 \\
c=c_{0}=\rho+\sqrt{\rho^{2}-1} & \text { if } & \rho<-1
\end{array}\right.
$$

where

$$
\rho \widehat{=} \frac{2 m_{1}+1}{2 m_{2}} .
$$

Note that $|\rho| \geq 1$, a fact that is a simple consequence of

$$
\sum_{k=0}^{\infty} k[x(k) \pm x(k-1)]^{2}=2 M_{1}(x)+M_{0}(x) \pm 2 M_{2}(x) \geq 0 .
$$

The minimum itself can be written

$$
B_{\text {min }}=\frac{\left[\left(m_{1}+1\right) \rho-m_{2}\right] c_{0}-0.5}{N\left(1-\rho c_{0}\right)} .
$$

For a fixed $b(-1<b<1)$, let $C_{F}=C_{F}\left(b ; m_{1}, m_{2}\right)$ denote the class of signals $f \in \ell^{2}[0, \infty)$ with given $m_{1}(b)=m_{1}$ and $m_{2}(b)=m_{2}$. There exist signals $f \in C_{F}$ that achieve the bound in (8); as a simple example, consider $C_{F}\left(0.5 ; \frac{11}{30},-\frac{1}{3}\right)$ : it is a standard exercise to show that $f(k) \widehat{=} 3 \psi_{0}(k, 0.5,-0.2)+\psi_{6}(k, 0.5,-0.2)$ is in this class. Clearly, the 6-term Kautz approximation using $\psi_{l}(k, 0.5,-0.2)$ is $\widetilde{f}(k) \hat{=} 3 \psi_{0}(k, 0.5,-0.2)$ with $q=0.1$ and $B=0.1$, whence $q=B$. Therefore, the bound in (8) is actually the maximum quadratic error for signals in $C_{F}$ and (9) gives the best $c$, in the sense of minimizing the maximum quadratic error, that can be obtained with the knowledge of the signal limited to $m_{1}(b)$ and $m_{2}(b)$.

Now, suppose that $m_{1}(b)$ and $m_{2}(b)$ are known for more than one value of $b$, say for $b \in S_{b}$ where $S_{b}$ represents a discrete or continuous subset of ]-1,1[. Since a proper choice of $b$ will result in the lowest $B_{\min }$, we have the following theorem.

Theorem 1: Let $b_{0}$ denote that value of $b \in S_{b}$ at which $B_{\min }$ is minimum and let $c_{0}$ be given by (9). Then, a pertinent choice for the pair of Kautz parameters is $\left(b_{0}, c_{0}\right)$, which yields $\left(B_{\min }\right)_{0}$.

Remark 1: The expression pertinent choice means that when the knowledge of the signal $f(k)$ under consideration is limited to a given limited set of moment values $m_{1}\left(b_{i}\right)$ and $m_{2}\left(b_{i}\right)$, the best choice of the parameters $b$ and $c$ is that which minimizes $B$. Notice that further improvment is usually possible using a standard unidimensional search algorithm to determine that value of $b$ which minimizes $B$.

Remark 2: Since parameters $b$ and $c$ are real the poles of Kautz functions are real or complex conjugate.

Remark 3: Notice that $b_{0}$ and $c_{0}$ do not depend on the number $L=2 N$ of functions to be used. Thus $b_{0}$ and $c_{0}$ can be computed in a first time and $L$, satisfying

$$
L \geq L_{\min }=2 \max \left(\frac{\left(M_{1}\left(g_{i}\right)+M_{0}\left(g_{i}\right)\right) c_{0}^{2}-2 c_{0} M_{2}\left(g_{i}\right)+M_{1}\left(g_{i}\right)}{\left(1-c_{0}^{2}\right) M_{0}\left(g_{i}\right)}\right) \quad(i=e, o)
$$

can be chosen afterwards: for instance, one can choose $L$ such that the upper bound $\left(B_{\min }\right)_{0}$ is small enough or such that the exact $q=1-\sum_{l=0}^{L-1} a_{l}^{2} /\|f\|^{2}$ is small enough.

\section{MOMENTS CALCULUS}

When $F(z)$ is a rational function, $G_{e}(z)$ and $G_{o}(z)$ can be obtained using equations (6)-(7) and the required moments can be computed directly in the z-domain [20]. However, in the case of experimental signals, the previous procedure is not suitable, so, in this section, we derive an alternative procedure using a relationship between the Kautz and the Laguerre functions.

Theorem 2: The functions $g_{e}(k, b)$ and $g_{o}(k, b)$ defined by $(4)$ and $(5)$ can be written

$$
\begin{gathered}
g_{e}(k, b)=\sum_{i=0}^{\infty} f(i) \psi_{2 k}(i, b, 0) \\
g_{o}(k, b)=\sum_{i=0}^{\infty} f(i) \psi_{2 k+1}(i, b, 0)
\end{gathered}
$$

where $\psi_{j}(i, b, 0)$ are the discrete Kautz functions with $c=0$. 
Proof: Using $a_{l}=\left\langle f, \psi_{l}\right\rangle$ and (4) in discrete-time domain, it follows

$$
g_{e}(k, b)=\sum_{i=0}^{\infty} f(i) \sum_{j=0}^{\infty} \phi_{j}(k, c) \psi_{2 j}(i, b, c) .
$$

Noting $H_{e}(z, k)$ the z-transform with respect to $i$ of $h_{e}(i, k) \widehat{=} \sum_{j=0}^{\infty} \phi_{j}(k, c) \psi_{2 j}(i, b, c)$, using (1) we deduce

$$
H_{e}(z, k)=\frac{z \sqrt{\left(1-c^{2}\right)\left(1-b^{2}\right)}}{z^{2}+b(c-1) z-c} \sum_{j=0}^{\infty} \phi_{j}(k, c)\left(\frac{-c z^{2}+b(c-1) z+1}{z^{2}+b(c-1) z-c}\right)^{j}
$$

where we recognize the z-transform with respect to $j$ of the Laguerre functions $\sum_{j=0}^{\infty} \phi_{j}(k, c) w^{-j}=\frac{w \sqrt{1-c^{2}}}{w+c}\left(\frac{1+c w}{w+c}\right)^{k}$ with the all-pass transformation $w=\frac{z^{2}+b(c-1) z-c}{-c z^{2}+b(c-1) z+1}$. Therefore after simplification it follows

$$
H_{e}(z, k)=z^{-(k+1)} \frac{z \sqrt{1-b^{2}}}{z-b}\left(\frac{1-b z}{z-b}\right)^{k}
$$

and using (3)

$$
h_{e}(i, k) \widehat{=} \sum_{j=0}^{\infty} \phi_{j}(k, c) \psi_{2 j}(i, b, c)=\phi_{k}(i-k-1, b)
$$

where $\phi_{k}(i-k-1, b)=0$ for $i<k+1$. $h_{e}(i, k)$ can also be written using the Kautz functions $(1)$ with $c=0$

$$
h_{e}(i, k) \widehat{=} \sum_{j=0}^{\infty} \phi_{j}(k, c) \psi_{2 j}(i, b, c)=\psi_{2 k}(i, b, 0)
$$

The last part of the theorem follows readily. The demonstration is similar for $g_{o}(k, b)$. We deduce that

$$
h_{o}(i, k) \widehat{=} \sum_{j=0}^{\infty} \phi_{j}(k, c) \psi_{2 j+1}(i, b, c)=\frac{1}{\sqrt{1-b^{2}}}\left[\phi_{k}(i-k, b)-b \phi_{k}(i-k-1, b)\right]
$$

or in terms of Kautz functions

$$
h_{o}(i, k) \widehat{=} \sum_{j=0}^{\infty} \phi_{j}(k, c) \psi_{2 j+1}(i, b, c)=\psi_{2 k+1}(i, b, 0)
$$

and then (11) follows.

\section{ILLUSTRATIVE EXAMPLE}

Consider the z-transform

$$
F(z)=\frac{10 z^{4}+12 z^{3}-10 z^{2}+8 z+1}{10 z^{4}-20 z^{3}+21 z^{2}-12 z+4}
$$

with the aim to derive a second-order approximation $(L=2 N=2$ Kautz functions). Letting for example $b=0.7,(6)$ and (7) yield

$$
\begin{aligned}
G_{e}(z) & =\frac{\sqrt{51} z}{4} \frac{6357 z^{3}+10213 z^{2}+6333 z+1497}{2612 z^{4}+5792 z^{3}+5407 z^{2}+2462 z+477} \\
G_{o}(z) & =\frac{1}{4} \frac{10448 z^{4}+21721 z^{3}+15681 z^{2}+5873 z+477}{2612 z^{4}+5792 z^{3}+5407 z^{2}+2462 z+477}
\end{aligned}
$$

Using one of the available techniques (e.g. [20]), the required normalized moments are computed as $\|f\|^{2}=29.824$, $m_{1}(0.7)=0.5481, m_{2}(0.7)=-0.8381$. From (9), the error bound is then minimized when $c=-0.500$. With $b=0.700$ and $c=-0.500$, the first and second coefficients of the Kautz expansion are $a_{0}=5.181$ and $a_{1}=0.769$ from which the exact normalized quadratic error is obtained as $q=8.008 \times 10^{-2}$.

The normalized moments $m_{1}$ and $m_{2}$ computed by repeating the procedure for $b=0.8$ and $b=0.9$ are shown in Table 1. For $b \in\{0.7,0.8,0.9\}$, the bound $B_{\min }$ is minimum if $b=0.8$; therefore, in agreement with theorem 1 , we select $b_{o}=0.8$ and $c_{o}=-0.650$, improving the normalized quadratic error, which becomes $q=q_{o}=2.449 \times 10^{-2}$. It is worth noting that $q_{o}$ obtained using limited knowledge of the signal (Table 1 ) is, for this example, very close to the best possible value $q_{o p t}=2.276 \times 10^{-2}$ that can be achieved with complete knowledge of the signal with $b=0.797$ and $c=-0.667$. 


\section{CONCLUSION}

An upper bound for the quadratic truncation error in discrete two-parameter Kautz approximation has been derived. It is a function of the free parameters and some moments relative to the function to be modeled. Minimization of this upper bound yields pertinent parameters that are very simple to compute. In the case of a limited number of expansion terms, this easy-to-use procedure, yields a proper choice of the pair of free parameters in discrete-time Kautz approximation. The method doesn't require an initially given order of approximation contrary to an optimal technique where the free parameters depend to this order of approximation. The results presented herein will enforce the interest in the use of Kautz models, which seem to have large potential in many applications in control theory and signal processing, where modeling of resonant systems is of importance.

\section{ACKNOWLEDGMENT}

The authors would like to thank the reviewers for their valuable comments and suggestions.

\section{REFERENCES}

[1] P. Lindskog and B. Walhberg, "Applications of Kautz models in system identification" IFAC 12th Triennal World Congress, Sydney, Australia, pp 41-44, 1993.

[2] B. Wahlberg, "System identification using Kautz models". IEEE Trans. on Automatic Control, vol 39, no 6, pp 1276-1282, june 1994.

[3] B. Wahlberg, and P.M.Mäkilä, "On approximation of stable linear dynamical systems using Laguerre and Kautz functions", Automatica, vol. 32, no 5, pp. 693-708, 1996.

[4] M.A. Masnadi-Shirazi, and N. Ahmed, "Laguerre approximation of non recursive discrete-time systems", Proc. IEEE Int. Conf. Acoust., Speech, Signal Processing, (Albuquerque, NM), apr. 3-6, pp. 1309-1312, 1990.

[5] M.A. Masnadi-Shirazi, and N. Ahmed, "Optimum Laguerre networks for a class of discrete-time systems", IEEE Trans. on Signal Processing, vol. 39, pp. 2104-2108, 1991.

[6] M.A. Masnadi-Shirazi, "Optimum synthesis of linear discrete-time systems using orthogonal Laguerre sequences", Ph. D. dissertation, Univ. New-Mexico, may 1990.

[7] T. Oliveira e Silva, "Optimality conditions for truncated Laguerre networks", IEEE Trans. on Signal Processing, vol. 42, no 9, pp. 2528-2530, sept. 1994.

[8] T. Oliveira e Silva, "On the determination of the optimal pole position of Laguerre filters", IEEE Trans. on Signal Processing, vol. 43, no 9, pp. 2079-2087, sept. 1995.

[9] T. Oliveira e Silva, "Optimality conditions for truncated Kautz networks with two periodically repeating complex conjugate poles", IEEE Trans. on Automatic Control, vol 40, no 2, pp 342-346, feb. 1995.

[10] A.C. Den Brinker, F.P.A. Benders, and T. Oliveira e Silva, "Optimality conditions for truncated Kautz series", IEEE Trans. on Circuits and Systems II: Analog and Digital Signal Processing, vol. 43, no2, pp. 117-122, feb. 1996.

[11] G.J. Clowes, "Choice of the time-scaling factor for linear system approximations using orthonormal Laguerre functions". IEEE Trans. on Automatic Control, vol. 10, pp 487-489, oct. 1965.

[12] T.W. Parks, "Choice of time scale in Laguerre approximations using signal measurements". IEEE Trans. on Automatic Control, vol. 16, pp 511-513, oct. 1971.

[13] Y. Fu and G.A. Dumont, "An optimum time scale for discrete Laguerre network", IEEE Trans. on Automat. Control, vol. 38, no 6, pp. 934-938, june 1993.

[14] Y. Fu and G.A. Dumont, "On determination of Laguerre filter pole through step or impulse response data", IFAC 12th Triennal World Congress, Sydney, Australia, pp. 35-39, 1993.

[15] N. Tanguy, P. Vilbé, and L.C. Calvez, "Optimum choice of free parameter in orthonormal approximations", IEEE Trans. on Automatic Control, vol. 40, no 10, pp. 1811-1813, 1995

[16] A.C. Den Brinker, and H.J.W Belt, "Optimal free parameters in orthonormal approximations", IEEE Trans. on Signal Processing, vol. 46, no 8, pp. 2081-2087, 1998.

[17] N. Tanguy, R. Morvan, P. Vilbé, and L.C. Calvez, "Improved method for optimum choice of free parameter in orthogonal approximations". IEEE Trans. on Signal Processing. vol. 47, no 9, pp 2576-2579, sept. 1999.

[18] R. Morvan, N. Tanguy, P. Vilbé, and L.C. Calvez, "Pertinent parameters for Kautz approximation". Electronics Letters, vol. 36, no 8, pp 769-771, april 2000.

[19] A.H. Gray Jr, JD. Markel, "A normalized digital filter structure", IEEE Trans. on Acoustics, Speech, and Signal Processing, Vol. ASSP-23, no 3, pp. 268-277, june 1975.

[20] A. Derrien, "Modélisation des systèmes linéaires à temps discret via un procédé d'orthogonalisation et un algorithme de Gauss-Newton" Thèse de doctorat, Université de Brest, France, 1994.

\begin{tabular}{|c|c|c|c|}
\hline$b$ & 0.7 & 0.8 & 0.9 \\
\hline$m_{1}(b)$ & 0.5481 & 0.8832 & 1.6462 \\
\hline$m_{2}(b)$ & -0.8381 & -1.2644 & -1.9772 \\
\hline$B_{\min }$ & 0.1294 & 0.0609 & 0.3350 \\
\hline$L_{\min }$ & 1.86 & 0.84 & 0.96 \\
\hline
\end{tabular}

Table 1: Normalized moments for $b=0.7,0.8,0.9$ 